\title{
Distinct topological properties in Ce monopnictides having correlated $f$ electrons: CeN vs. CeBi
}

\author{
Dong-Choon Ryu, ${ }^{1, *}$ Junwon Kim $\odot,{ }^{1,}{ }^{*}$ Kyoo Kim $\odot,{ }^{1,2}$ Chang-Jong Kang $\odot,{ }^{1, \dagger}$ J. D. Denlinger, ${ }^{3}$ and B. I. Min $\oplus^{1, \ddagger}$ \\ ${ }^{1}$ Department of Physics, Pohang University of Science and Technology, Pohang 37673, Korea \\ ${ }^{2}$ MPPC Pohang University of Science and Technology, Pohang 37673, Korea \\ ${ }^{3}$ Advanced Light Source, Lawrence Berkeley Laboratory, Berkeley, California 94720, USA
}

(Received 6 September 2019; accepted 4 March 2020; published 18 March 2020)

\begin{abstract}
The hitherto-studied topological nature in Ce monopnictides $(\mathrm{Ce} P n)$ has been discussed based solely on their $p-d$ band inversion, despite the existence of $f$ electrons. Interestingly, however, we have found that CeN, the lightest $\mathrm{Ce} P n$, has nontrivial $Z_{2}$ topology originating from $f-d$ band inversion. Through density-functional theory and dynamical mean-field theory calculations, we have demonstrated that, among the correlated $f$ electron systems of $\mathrm{Ce} P n$, the lightest $\mathrm{CeN}$ is a coherent narrow $f$-band system having an $f$ - $d$ band-inverted topological Kondo insulator nature, whereas the heaviest $\mathrm{CeBi}$ is a strongly localized $f$-electron system having a conventional $p$ - $d$ band-inverted topological insulator nature. For CeN, topological surface states (TSSs) are identified clearly on three different (001), (110), and (111) surfaces. For CeBi, however, TSSs are identified only on the (111) surface. Furthermore, intriguing topological-crystalline insulator-type TSSs are identified on the (110) surfaces of both $\mathrm{CeN}$ and $\mathrm{CeBi}$.
\end{abstract}

DOI: 10.1103/PhysRevResearch.2.012069

Ce monopnictides, $\mathrm{Ce} P n$, have attracted a great deal of renewed attention as potential candidates of nontrivial $Z_{2}$ topological matter [1-5]. Alidoust et al. [1] reported first that $\mathrm{CeSb}$ and $\mathrm{CeBi}$ would have a topological-insulator (TI) nature originating from $\mathrm{Sb}(\mathrm{Bi}) p-\mathrm{Ce} 5 d$ band inversion. Using vacuum-ultraviolet (VUV) angle-resolved photoemission spectroscopy (ARPES), they observed seemingly Dirac-conelike dispersions on the (001) surfaces of $\mathrm{CeSb}$ and $\mathrm{CeBi}$. Subsequent studies, however, reported contradictory results, particularly on the topological nature of $\mathrm{CeSb}[2,3]$. Based on bulk-sensitive soft x-ray ARPES and density-functional theory (DFT) calculations, Oinuma et al. [2] reported that $\mathrm{CeSb}$ is merely a topologically trivial semimetal without $p$ - $d$ band inversion, and that the Dirac-cone-like states observed in VUV ARPES are not of a topological origin. Kuroda et al. [3] also investigated the bulk electronic structures of $\mathrm{CePn}$ using bulk-sensitive soft x-ray ARPES, and reported that only the heaviest $\mathrm{CeBi}$ has $p$ - $d$ band inversion, while other $\mathrm{CePn}$ $(P n=\mathrm{P}, \mathrm{As}$, and $\mathrm{Sb})$ just have trivial gaps between $p$ and $d$ bands.

Even for $\mathrm{CeBi}$, however, no clear Dirac-band crossings were observed on its (001) surface [1-4]. It is because the topological surface states (TSSs) expected at $\bar{\Gamma}$ of the surface Brillouin zone (BZ) [see Fig. 1(a)] are buried under the bulk-

\footnotetext{
*These authors contributed equally to this work.

†Present address: Department of Physics and Astronomy, Rutgers University, Piscataway, New Jersey 08854, USA.

‡bimin@ postech.ac.kr

Published by the American Physical Society under the terms of the Creative Commons Attribution 4.0 International license. Further distribution of this work must maintain attribution to the author(s) and the published article's title, journal citation, and DOI.
}

projected $\mathrm{Bi} 6 p$ band. In addition, the double Dirac cones anticipated to be at $\bar{M}$ in Fig. 1(a), which are induced from band inversions at two nonequivalent bulk $X$ points, would be gapped by their mutual hybridization, so as to lose their topological signature revealing trivial Rashba-type surface states $[4,5]$.

Note that the topological nature for $\mathrm{Ce} P n$ has been investigated based solely on $p$ - $d$ band inversion because $f$ electrons in $\mathrm{CeSb}$ and $\mathrm{CeBi}$ are assumed to be inactive due to their localized nature [1-4]. This assumption, however, is no longer valid when the $\mathrm{Ce}-\mathrm{Ce}$ distances become closer, as in $\mathrm{CeN}$ [see Fig. 1(b)]. The X-ray photoemission spectroscopy (XPS) study by Baer et al. [6] showed that $\mathrm{CeN}$ has a clear $4 f$ quasiparticle band near the Fermi level $\left(E_{\mathrm{F}}\right)$, exhibiting a mixed-valent behavior of $\mathrm{Ce}$ ions [7-10]. Thus $\mathrm{CeN}$ is expected to have markedly different physical properties from other $\mathrm{CePn}$. For example, $\mathrm{CeP}$, the second lightest $\mathrm{Ce} P n$, already has an integer valence state of $\mathrm{Ce}$ ions and the magnetic ground state, behaving as $\gamma$-Ce-like [see Fig. 1(b)]. Previous DFT [11-15] and dynamical mean-field theory (DMFT) [16-18] calculations for $\mathrm{CeN}$ also showed the $f$-band formation and the mixedvalent behavior of Ce ions. It is thus generally accepted that $f$ electrons in $\mathrm{CeN}$ are delocalized, so that $\mathrm{CeN}$ behaves as an $\alpha$-Ce-like narrow $f$-band metallic system [6-19].

In $\mathrm{CeN}$, the coherent $f$ band is supposed to be already formed at ambient pressure through the temperature $(T)$ dependent Kondo effect, which is absent in other heavier $\mathrm{Ce} P n$. Then the coherent $f$ band in $\mathrm{CeN}$ is expected to produce $f$ - $d$ band inversion, which leads to a nontrivial topology of the topological Kondo insulator (TKI) type [20,21]. In fact, the nontrivial topology due to the $f$ - $d$ band inversion in a facecentered-cubic (fcc) system was discussed previously for a golden phase of SmS $(g-\mathrm{SmS})$ [22-24], which is isostructural to $\mathrm{CePn}$. This suggests that the TSSs would possibly emerge in $\mathrm{CeN}$ through $f$ - $d$ band inversion, quite distinctly from 

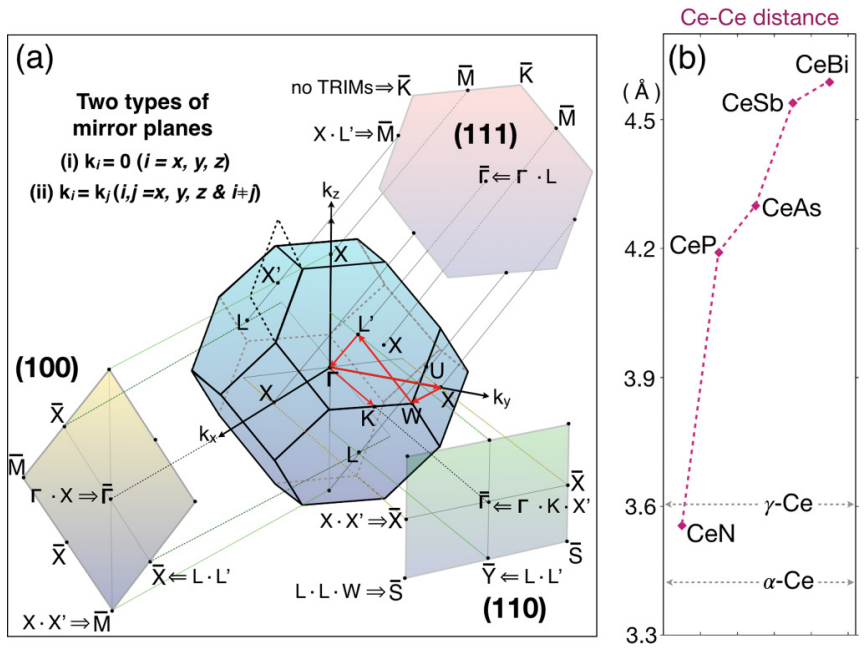

FIG. 1. (a) Bulk and surface BZ of fcc CePn. (b) Ce-Ce distances of $\mathrm{Ce} P n$ together with those of $\alpha$-Ce and $\gamma$-Ce (see also Fig. S1 in the Supplemental Material [28]).

those in other $\mathrm{CePn}$. It is thus imperative to investigate the electronic structures and the topological properties of $\mathrm{CeN}$ in comparison with those of other $\mathrm{Ce} P n$.

In this Rapid Communication, employing the DFT and DMFT band methods [25], we demonstrate that the coherent $f$ band and the consequent hybridization gap are formed in $\mathrm{CeN}$, which produces the $f-d$ band inversion. Then, based on the surface band calculations, we show that there indeed exist TSSs on three different (001), (110), and (111) surfaces, which are not buried in the bulk continuum states, confirming the TKI nature in CeN. Further, we have compared the electronic structures and the topological properties between $\mathrm{CeN}$ and $\mathrm{CeBi}$, which have delocalized and localized $f$ electrons, respectively.

We have performed the band structure calculations for $\mathrm{CeN}$ and CeBi by employing both DFT and DFT+DMFT [26,27]. Surface band structures are obtained based on the Wannierized tight-binding (TB) Hamiltonian. Computational details are provided in the Supplemental Material [28].


Figure 2 shows the DFT+DMFT band structures and Fermi surfaces (FSs) of $\mathrm{CeN}$ and CeBi. Neither the band structure with spectral intensities nor the FS data were reported in previous DMFT calculations for CeN [16-18]. As the $\mathrm{Ce}-\mathrm{Ce}$ distance is getting closer, the $4 f$ electrons become delocalized. For this reason, the quasiparticle renormalization factor $Z_{\mathrm{F}}$ for the $4 f$ electrons is expected to increase with decreasing atomic number of $P n$ in $\mathrm{Ce} P n$ series. According to the DMFT calculations, $Z_{\mathrm{F}}$ values for $\mathrm{Ce} P n$ turn out to be negligible (less than 0.06 even for $\mathrm{CeP}$ ) except for $\mathrm{CeN}$, which has $Z_{\mathrm{F}} \approx 0.4$ (see Fig. S1 in the Supplemental Material [28]). This non-negligible $Z_{\mathrm{F}}$ value in $\mathrm{CeN}$ gives rise to clearly different low-energy spectra from those of other $\mathrm{Ce} P n$. That is, CeN has an almost coherent $4 f$ quasiparticle band near $E_{\mathrm{F}}$, which is absent in other $\mathrm{Ce} P n$. This difference is highlighted in Figs. 2(a) and 2(c), which compares the DMFT band structures between $\mathrm{CeN}$ and $\mathrm{CeBi}$ at $T=290 \mathrm{~K}$. It is shown that most of the $4 f$ spectral weights in $\mathrm{CeN}$ are located around $E_{\mathrm{F}}$ in the form of a Kondo coherent band. In contrast, $4 f$ spectral weights in $\mathrm{CeBi}$ are negligible near $E_{\mathrm{F}}$, but are located incoherently around the upper and lower Hubbardband regions together with complex multiplet structures in between, and so CeBi exhibits a simple semimetallic band structure as LaBi $[29,30]$.

Whether or not the $f$ electrons participate in the valence state is well reflected in the FS topologies in Figs. 2(b) and 2(d). For CeN, as shown in Fig. 2(b), the DFT-FS has good agreement with the DMFT-FS at $T=290 \mathrm{~K}$, but is a bit different from the DMFT-FS at $T=500 \mathrm{~K}$. Note that, in the DFT, the $f$ electrons are treated as coherent valence electrons, and so this agreement implies that the $4 f$ electrons in $\mathrm{CeN}$ are forming a quasiparticle band with decreasing $T$ from 500 to $290 \mathrm{~K}$, and establish a coherent band already at around room temperature [31]. On the other hand, for $\mathrm{CeBi}$, a great similarity is observed in Fig. 2(d) between the DMFTFSs at $T=100$ and $290 \mathrm{~K}$ and the DFT-" $f$-opencore"-FS. Since the " $f$-opencore" calculation excludes the $f$ electrons from the valence states, this similarity implies that the $4 f$ electrons in $\mathrm{CeBi}$ are strongly localized without showing any $T$ dependence and so do not contribute to the FS [32]. Even at lower $T$, the $4 f$ electrons in CeBi will not be delocalized

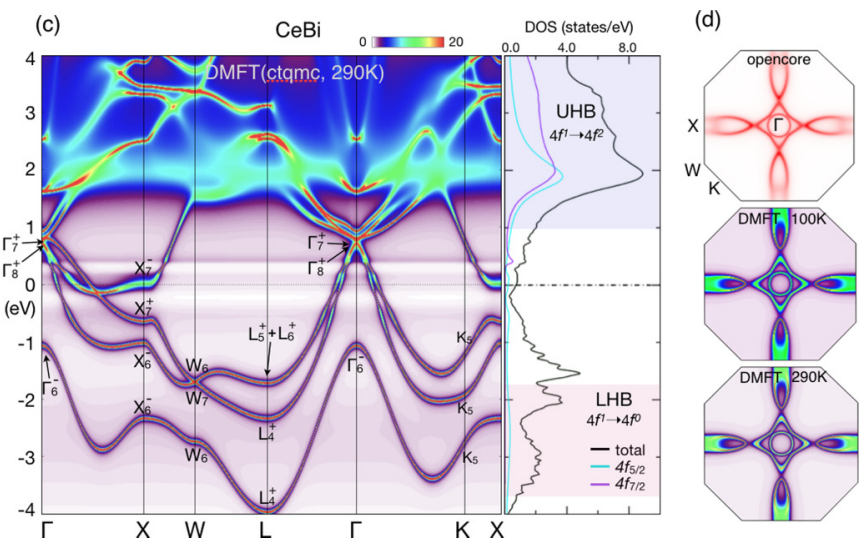

FIG. 2. (a) DFT+DMFT band structure of $\mathrm{CeN}$ at the ambient pressure and $T=290 \mathrm{~K}$. Irreducible representation of each band is provided. (b) Comparison of the FSs of CeN obtained by DFT and DFT+DMFT at $T=290$ and $580 \mathrm{~K}$. (c) DFT+DMFT band structure of CeBi at the ambient pressure and $T=290 \mathrm{~K}$. (d) Comparison of the FSs of CeBi obtained by DFT-opencore and DFT+DMFT at $T=100$ and $290 \mathrm{~K}$. In the DFT-opencore method, $4 f$ electrons are treated as core electrons. For the DMFT calculations, we used $U=5.5 \mathrm{eV}$ and $J=0.68 \mathrm{eV}$. 

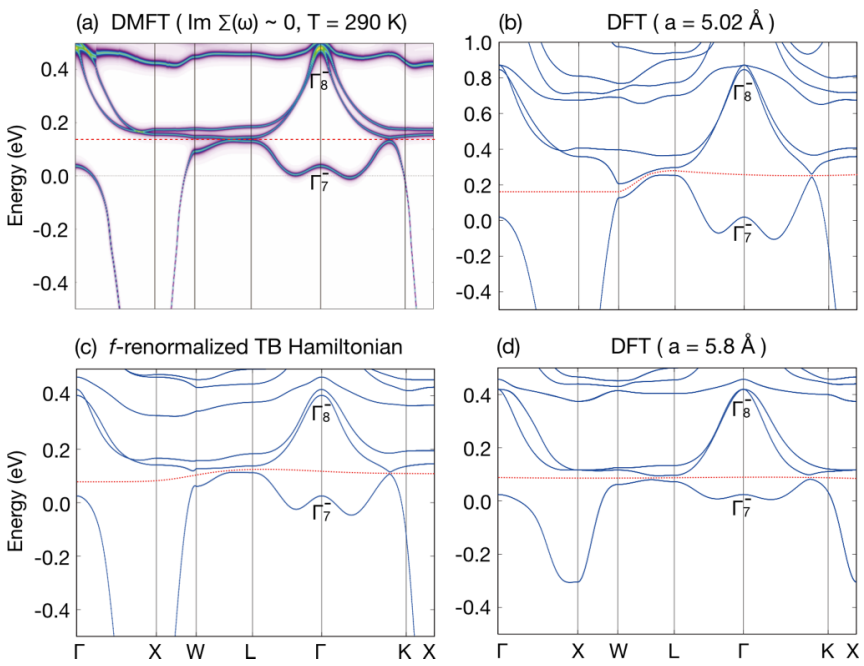

FIG. 3. Amplified bulk band structures of $\mathrm{CeN}$ near $E_{\mathrm{F}}$, obtained by (a) DMFT calculation at $T=290 \mathrm{~K}$ by setting the imaginary part of the self-energy zero. (b) Scheme (i): Ordinary DFT calculation ( $a=5.02 \AA$ ). (c) Scheme (ii): $f$-renormalized TB Hamiltonian. (d) Scheme (iii): DFT calculation with extended volume ( $a=5.80 \AA$ A $)$. Red lines denote fictitious chemical potentials inside the gap.

because they rather become magnetically polarized at low $T$. These results suggest that the low-energy physics in $\mathrm{CeN}$ can be described properly by the DFT, as previously reported for representative Kondo insulator systems, such as $\mathrm{SmB}_{6}$ [33] and $g$-SmS $[23,24]$, while that in CeBi by the DFT-" $f$ opencore" calculations as hitherto reported (see Fig. S2 in the Supplemental Material [28]).

The most remarkable in the DMFT band structure for $\mathrm{CeN}$ in Fig. 2(a) is its topological property resulting from the $f-d$ band inversion at $X$. The amplified DMFT band structure near $E_{\mathrm{F}}$ in Fig. 3(a) clearly shows a direct-gap opening in the full BZ between the upper $\Gamma_{8}^{-}$and the lower $\Gamma_{7}^{-}$band, even though the gap is small [34] and located above $E_{\mathrm{F}}$. Then, assuming a fictitious chemical potential (red line), the topological $Z_{2}$ index can be determined. As shown in Table S1 in the Supplemental Material [28], we have obtained $Z_{2}=1$, suggesting the nontrivial topological nature of $\mathrm{CeN}$. Meanwhile, for $\mathrm{CeBi}$, there is no $f$ - $d$ band inversion because of the absence of a coherent Ce $4 f$ band. But it is seen in Fig. 2(c) that there is a $p$ - $d$ band inversion at $X$ between $\Gamma_{7}^{-}$of $\mathrm{Bi} 6 p$ and $\Gamma_{7}^{+}$of Ce $5 d$ bands, producing nontrivial topology. The corresponding $p-d$ band inversion will not be operative in $\mathrm{CeN}$, as in $\mathrm{CeP}-\mathrm{CeSb}$, when the modified Becke-Johnson (mBJ) exchange potential is employed (see Fig. S3 in the Supplemental Material [28]). Starting from these bulk topological properties, we explore the TSSs in CeN and CeBi by carrying out surface slab calculations with Wannierized TB Hamiltonians.

Even though DFT-FS is close to DMFT-FS for CeN in Fig. 2(b), the overall DFT band structure in Fig. 3(b) is different from the DMFT band structure in Fig. 3(a), especially in the Ce $4 f$ bandwidth. Due to the overestimated $4 f$ bandwidth, the DFT does not produce a full direct gap, unlike the DMFT. Then the TSSs derived based on the DFT band structure will likely be merged into the bulk continuum states [see Figs. S4(a) and S4(b) in the Supplemental Material [28]). Hence, for better visibility of the TSSs, we have devised two more schemes to simulate the DMFT band in Fig. 3(a) in addition to the pure DFT scheme (i). In scheme (ii), we rescale the $f$-electron hopping parameters in the Wannierized TB Hamiltonian obtained from the pure DFT band by the quasiparticle renormalization factor $Z_{\mathrm{F}}$. Then the DFT $f-f$ and $f-c$ ( $c$ : conduction electrons) hopping parameters are reduced by $Z_{\mathrm{F}}$ and $\sqrt{Z_{\mathrm{F}}}$, respectively. We have found that $Z_{\mathrm{F}} \approx 0.4$, obtained from the DMFT calculations in Fig. S1, gives the band structure in Fig. 3(c) that is quite similar to the DMFT band structure. In scheme (iii), we obtain the Wannierized TB Hamiltonian from the DFT calculation for $\mathrm{CeN}$ with an extended volume $\left(V / V_{0}=1.54 ; a=5.8 \AA\right)$. As shown in Fig. 3, both schemes (ii) and (iii) reproduce low-energy DMFT band structures much better than the pure DFT scheme (i). It is notable that all three band structures in Figs. 3(b)-3(d) have the same topological invariants, such as $Z_{2}=1$ and the mirror Chern number $(\mathrm{MCN})$ set of $\left(C_{0}, C_{d}\right)=(-1,-1)$ (see Fig. S5 in the Supplemental Material [28]), and so all three schemes are expected to yield surface states having essentially the same topological nature. So, in the following, we adopt scheme (ii) to discuss the TSSs in CeN. The TSSs from the pure DFT scheme (i) and scheme (iii) are also provided in Fig. S4 in the Supplemental Material [28].

The upper panel of Fig. 4 displays the surface band structures on three different surfaces of CeN. On the (001) surface of a fcc lattice, as shown in Fig. 1(a), $X$ and $\Gamma$ in the bulk BZ are projected onto $\bar{\Gamma}$ in the surface BZ, while two $X$ points are projected onto $\bar{M}$. Accordingly, a single Dirac cone is expected to exist at $\bar{\Gamma}$, while double Dirac cones at $\bar{M}$. Indeed, on the (001) surface of CeN in Fig. 4(a), the TSSs of Dirac cone type at $\bar{\Gamma}$ are clearly seen at $E \approx 0.13 \mathrm{eV}$, even though they are intertwined intricately in the gap region. On the other hand, no feature of double Dirac cones is seen at $\bar{M}$. They are expected to be gapped through mutual hybridization, exhibiting only the lower Dirac cone at $\bar{M}$ in the gap region. The topological nature of TSSs at $\bar{\Gamma}$ is checked by the spin textures on the $\bar{\Gamma}$-centered constant-energy surfaces in Fig. 4(d), which manifests unique spin-helical structures of the TSSs of Dirac cone type.

On the (111) surface of $\mathrm{CeN}$, a single Dirac cone is expected at $\bar{M}$, and indeed the TSSs at $\bar{M}$ are evident at $E \approx 0.14 \mathrm{eV}$ on both the $\mathrm{Ce}$ - and N-terminated (111) surface band structures in Figs. 4(b) and S6(a). On the (110) surface, $X$ and $\Gamma$ are projected onto $\bar{\Gamma}$, and two $X$ are projected onto $\bar{X}$. So a single Dirac cone is expected at $\bar{\Gamma}$, and double Dirac cones at $\bar{X}$. In Fig. 4(c), on the (110) surface of CeN, the TSSs at $\bar{\Gamma}$ are clearly seen along $\bar{\Gamma}-\bar{Y}$ but barely seen along $\bar{\Gamma}-\bar{X}$ at $E \approx 0.13 \mathrm{eV}$, being mostly buried in the bulk continuum. The double Dirac cones at $\bar{X}$ seem to be gapped along $\bar{S}-\bar{X}$. But whether or not the double Dirac cones along $\bar{X}-\bar{\Gamma}$ exhibit the band crossing of a topological-crystalline insulator (TCI) type is hardly identified, because the tails of the surface states are buried in the bulk continuum. This point is discussed more below in describing the TSSs on the (110) surface of CeBi.

In the lower panel of Fig. 4, the surface band structures of $\mathrm{CeBi}$ are presented. We have checked that the DFT-" $f$ opencore" bulk band structure of $\mathrm{CeBi}$ produces the nontrivial MCN set of $\left(C_{0}, C_{d}\right)=(1,1)$, which can be considered as the 


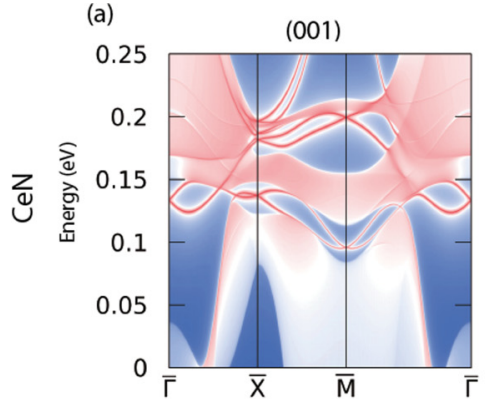

(e)

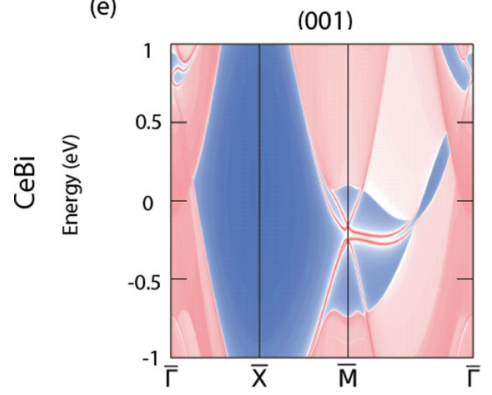

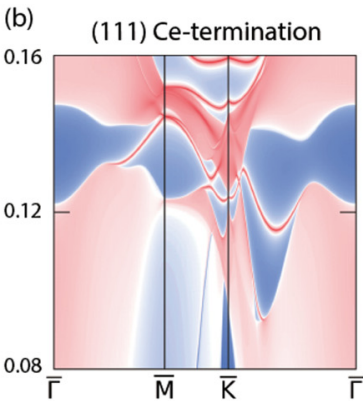

(f)



(c)

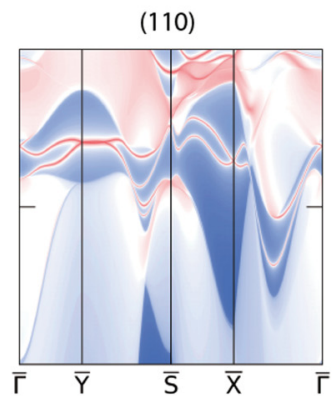

(g)

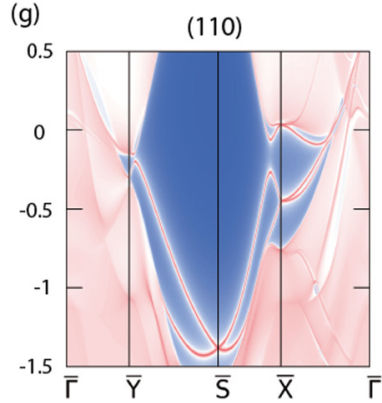

(d)

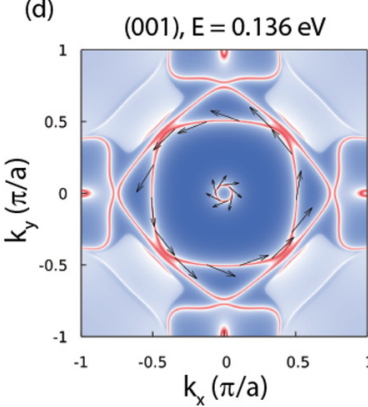

(h)

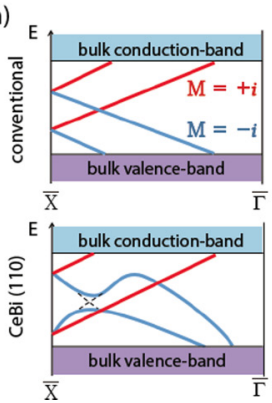

bulk

FIG. 4. Upper panel: (a)-(c) Surface band structures on different surfaces of CeN obtained from the slab calculation based on the $f$ renormalized Wannier Hamiltonian. (d) The constant-energy $(E=0.136 \mathrm{eV})$ surfaces of its $(001)$ surface. Opposite spin helicities (in black arrows) of the TSSs are manifested along two $\bar{\Gamma}$-centered energy surfaces. Lower panel: (e)-(g) Surface band structures on different surfaces of CeBi. (h) Schematic diagrams for the TSSs of the conventional TCI (upper) and CeBi (110) (lower). For both CeN and CeBi, the TSSs of single Dirac cones emerge on their (001) and (111) surfaces at $\bar{\Gamma}$ and $\bar{M}$, respectively, while the TSSs of intriguing TCI-type double Dirac cones appear on their (110) surfaces at $\bar{X}$.

same sort of MCN set of CeN up to $(\hat{M} \rightarrow-\hat{M})$ (see Fig. S5 in the Supplemental Material [28]), and so the topological nature of TSSs is expected to be the same between the two, despite the difference in the band-inversion type. As mentioned earlier, on the (001) surface of CeBi in Fig. 4(e), the expected TSSs at $\bar{\Gamma}$ are completely buried in the bulk continuum, and the double Dirac cones anticipated at $\bar{M}$ are gapped to reveal Rashba-type surface states [1,3]. By contrast, on the (111) surfaces in Figs. 4(f) and S6(b), the TSSs of TI Dirac cone type at $\bar{M}$ are readily noticeable at $E \approx-0.5 \mathrm{eV}$ for both Ce and Bi surface terminations, which span the direct bulk gap region.

On the other hand, on the (110) surface of $\mathrm{CeBi}$ in Fig. 4(g), the expected TSSs at $\bar{\Gamma}$ are seen to be completely buried in the bulk continuum, while the anticipated double Dirac cones at $\bar{X}$ show an unusual topological feature. The surface states from the double Dirac cones along $\bar{S}-\bar{X}$ are clearly gapped, but those along $\bar{X}-\bar{\Gamma}$ seem to have band crossings of TCI type. In fact, the analysis based on the mirror eigenvalues in Fig. S7 indicates that there would be double band crossings along $\bar{X}-\bar{\Gamma}$, which is quite distinct from just a single band crossing in conventional TCIs. This intriguing TCI feature arises from the reversed band order in the lower double Dirac cone (see Fig. 4(h) and Fig. S7). This TCI feature in $\mathrm{CeBi}$ is suggestive of an existence of a similar TCI feature on the (110) surface of CeN in Fig. 4(c).

We have thus found that $\mathrm{CeN}$ is an $f-d$ band-inverted TKI system, whereas CeBi is a conventional $p-d$ band-inverted TI system. For both $\mathrm{CeN}$ and $\mathrm{CeBi}$, the TSSs of single Dirac cones emerge on their (001) and (111) surfaces at $\bar{\Gamma}$ and $\bar{M}$, respectively, while the TSSs of intriguing TCI-type double Dirac cones appear on their (110) surfaces at $\bar{X}$. Among those, the TSSs on the (111) surfaces are the most prominent in the bulk-gap regions for both $\mathrm{CeN}$ and $\mathrm{CeBi}$, and so it is highly desirable to probe experimentally the TSSs on their (111) surfaces to verify the distinct topological properties in Ce monopnictides.

As a possible experimental probe, ARPES for alkali-metaldoped $\mathrm{CeN}$ or inverse ARPES for a normal $\mathrm{CeN}$ sample could be utilized to detect the unoccupied TSSs located above $E_{\mathrm{F}}$ $[35,36]$. It is noteworthy that CeN would be a TKI system having identifiable Dirac points located inside the large gap region. This feature in $\mathrm{CeN}$ is in stark contrast to that in $\mathrm{SmB}_{6}$, for which the Dirac point is known to be buried under the bulk-projected bands [33], and so is hardly detected in ARPES. The present systematic study on $\mathrm{CePn}$ suggests that strongly correlated systems with less localized $f$ electrons would be better TKI candidates, because they are expected to have larger Kondo gaps and higher $T_{\text {coh. }}$. In this respect, $5 f$-electron systems would be more promising for alternate TKIs than $4 f$-electron systems.

This work was supported by the NRF (Grants No. 2017R1A2B4005175, No. 2018R1A6A3A01013431, and No. 2016R1D1A1B02008461), Max-Plank POSTECH/KOREA Research Initiative (No. 2016K1A4A4A01922028), the POSTECH BSRI Grant, and the KISTI supercomputing center (Grant No. KSC-2018-CRE-0064). 
[1] N. Alidoust, A. Alexandradinata, S.-Y. Xu, I. Belopolski, S. K. Kushwaha, M. Zeng, M. Neupane, G. Bian, C. Liu, D. S. Sanchez, P. P. Shibayev, H. Zheng, L. Fu, A. Bansil, H. Lin, R. J. Cava, and M. Z. Hasan, arXiv:1604.08571.

[2] H. Oinuma, S. Souma, D. Takane, T. Nakamura, K. Nakayama, T. Mitsuhashi, K. Horiba, H. Kumigashira, M. Yoshida, A. Ochiai, T. Takahashi, and T. Sato, Phys. Rev. B 96, 041120(R) (2017).

[3] K. Kuroda, M. Ochi, H. S. Suzuki, M. Hirayama, M. Nakayama, R. Noguchi, C. Bareille, S. Akebi, S. Kunisada, T. Muro, M. D. Watson, H. Kitazawa, Y. Haga, T. K. Kim, M. Hoesch, S. Shin, R. Arita, and T. Kondo, Phys. Rev. Lett. 120, 086402 (2018).

[4] P. Li, Z. Wu, F. Wu, C. Cao, C. Guo, Y. Wu, Y. Liu, Z. Sun, C.-M. Cheng, D.-S. Lin, F. Steglich, H. Yuan, T.-C. Chiang, and Y. Liu, Phys. Rev. B 98, 085103 (2018).

[5] H. Oinuma, S. Souma, K. Nakayama, K. Horiba, H. Kumigashira, M. Yoshida, A. Ochiai, T. Takahashi, and T. Sato, Phys. Rev. B 100, 125122 (2019).

[6] Y. Baer, R. Hauger, Ch. Zürcher, M. Campagna, and G. K. Wertheim, Phys. Rev. B 18, 4433 (1978).

[7] W. Gudat, R. Rosei, J. H. Weaver, E. Kaldis, and F. Hulliger, Solid State Commun. 41, 37 (1982).

[8] E. Wuilloud, B. Delley, W.-D. Schneider, and Y. Baer, J. Magn. Magn. Mater. 47, 197 (1985).

[9] A. Fujimori and J. H. Weaver, Phys. Rev. B 31, 6345 (1985).

[10] F. Patthey, S. Cattarinussi, W.-D. Schneider, and Y. Baer, Europhys. Lett. 2, 883 (1986).

[11] W. E. Pickett and B. M. Klein, J. Less-Common Met. 93, 219 (1983).

[12] S. K. De and S. Chatterjee, J. Phys. C: Solid State Phys. 21, 3261 (1988).

[13] A. Delin, P. M. Oppeneer, M. S. S. Brooks, T. Kraft, J. M. Wills, B. Johansson, and O. Eriksson, Phys. Rev. B 55, R10173 (1997).

[14] G. A. Landrum, R. Dronskowski, R. Niewa, and F. J. Disalvo, Chem. - Eur. J. 5, 515 (1999).

[15] P. Larson, W. R. L. Lambrecht, A. Chantis, and M. van Schilfgaarde, Phys. Rev. B 75, 045114 (2007).

[16] J. Laegsgaard and A. Svane, Phys. Rev. B 58, 12817 (1998).

[17] O. Sakai and Y. Shimizu, J. Phys. Soc. Jpn. 76, 044707 (2007).

[18] M. S. Litsarev, I. Di Marco, P. Thunström, and O. Eriksson, Phys. Rev. B 86, 115116 (2012).

[19] The Ce-Ce distance in CeN is larger than that in $\alpha$-Ce, but the Ce $f$ bandwidth is wider due to strong Ce $f-\mathrm{N} p$ hybridization.

[20] M. Dzero, K. Sun, V. Galitski, and P. Coleman, Phys. Rev. Lett. 104, 106408 (2010).

[21] M. Dzero, K. Sun, P. Coleman, and V. Galitski, Phys. Rev. B 85, 045130 (2012).

[22] Z. Li, J. Li, P. Blaha, and N. Kioussis, Phys. Rev. B 89, 121117(R) (2014).

[23] C.-J. Kang, H. C. Choi, K. Kim, and B. I. Min, Phys. Rev. Lett. 114, 166404 (2015).

[24] C.-J. Kang, D. C. Ryu, J. W. Kim, K. Kim, J.-S. Kang, J. D. Denlinger, G. Kotliar, and B. I. Min, Phys. Rev. Materials 3, 081201(R) (2019).

[25] A. Georges, G. Kotliar, W. Krauth, and M. J. Rozenberg, Rev. Mod. Phys. 68, 13 (1996).
[26] K. Haule, C.-H. Yee, and K. Kim, Phys. Rev. B 81, 195107 (2010).

[27] G. Kotliar, S. Y. Savrasov, K. Haule, V. S. Oudovenko, O. Parcollet, and C. A. Marianetti, Rev. Mod. Phys. 78, 865 (2006).

[28] See Supplemental Material at http://link.aps.org/supplemental/ 10.1103/PhysRevResearch.2.012069 for (i) the computational details, (ii) $Z_{2}$ index of $\mathrm{CeN}$, (iii) the quasiparticle renormalization factor $Z_{\mathrm{F}}$, (iv) DMFT vs DFT FSs of CeN and CeBi, (v) bulk band structure of $\mathrm{CeN}$ by using the $\mathrm{mBJ}$ potential, (vi) robustness of the Kondo gap, (vii) surface band structures of $\mathrm{CeN}$ from the DFT at experimental and extended volumes, (viii) MCNs for $\mathrm{CeN}$ and $\mathrm{CeBi}$, (ix) (111) surface band structures of $\mathrm{CeN}$ with $\mathrm{N}$ termination and $\mathrm{CeBi}$ with $\mathrm{Bi}$ termination, and (x) mirror eigenvalue-projected (110) surface band structures of CeBi, which includes Refs. [37-48].

[29] Y. Wu, T. Kong, L.-L. Wang, D. D. Johnson, D. Mou, L. Huang, B. Schrunk, S. L. Bud'ko, P. C. Canfield, and A. Kaminski, Phys. Rev. B 94, 081108(R) (2016).

[30] J. Nayak, S.-C. Wu, N. Kumar, C. Shekhar, S. Singh, J. Fink, E. E. D. Rienks, G. H. Fecher, S. S. P. Parkin, B. Yan, and C. Felser, Nat. Commun. 8, 13942 (2017).

[31] J. Danan, C. de Novion, and R. Lallement, Solid State Commun. 7, 1103 (1969).

[32] T. Kasuya, O. Sakai, J. Tanaka, H. Kitazawa, and T. Suzuki, J. Magn. Magn. Mater. 63, 9 (1987).

[33] J.-W. Kim, K. Kim, C.-J. Kang, S.-R. Kim, H. C. Choi, J.-S Kang, J. D. Denlinger, and B. I. Min, Phys. Rev. B 90, 075131 (2014).

[34] The smallest gap $(\sim 10 \mathrm{meV})$ is located in between $W$ and $L$.

[35] C.-J. Kang, J. D. Denlinger, J. W. Allen, C.-H. Min, F. Reinert, B. Y. Kang, B. K. Cho, J.-S. Kang, J. H. Shim, and B. I. Min, Phys. Rev. Lett. 116, 116401 (2016).

[36] J. A. Sobota, S.-L. Yang, A. F. Kemper, J. J. Lee, F. T. Schmitt, W. Li, R. G. Moore, J. G. Analytis, I. R. Fisher, P. S. Kirchmann, T. P. Devereaux, and Z.-X. Shen, Phys. Rev. Lett. 111, 136802 (2013).

[37] G. Kresse and J. Furthmüller, Phys. Rev. B 54, 11169 (1996); Comput. Mater. Sci. 6, 15 (1996).

[38] P. Blaha, K. Schwarz, G. K. H. Madsen, D. Kvasnicka, and J. Luitz, WIEN2K (Karlheinz Schwarz, Technische Universitat Wien, Austria, 2001).

[39] K. Haule, Phys. Rev. B 75, 155113 (2007).

[40] P. Sémon, C. H. Yee, K. Haule, and A.-M. S. Tremblay, Phys. Rev. B 90, 075149 (2014).

[41] A. A. Mostofi, J. R. Yates, G. Pizzi, Y. S. Lee, I. Souza, D. Vanderbilt, and N. Marzari, Comput. Phys. Commun. 185, 2309 (2014).

[42] Q. S. Wu, S. N. Zhang, H.-F. Song, M. Troyer, and A. A. Soluyanov, Comput. Phys. Commun. 224, 405 (2018).

[43] G. L. Olcese, J. Phys. F: Met. Phys. 9, 569 (1979).

[44] J. M. Léger, K. Oki, J. Rossat-Mignod, and O. Vogt, J. Phys. (Paris) 46, 889 (1985).

[45] X. Duan, F. Wu, J. Chen, P. Zhang, Y. Liu, H. Yuan, and C. Cao, Commun. Phys. 1, 71 (2018)

[46] P. Wachter and N. D. Zhigadlo, Results Phys. 3, 235 (2013).

[47] R. Yu, X. L. Qi, A. Bernevig, Z. Fang, and X. Dai, Phys. Rev. B 84, 075119 (2011).

[48] A. A. Soluyanov and D. Vanderbilt, Phys. Rev. B 83, 235401 (2011). 